\title{
Understanding How Pair Programming Actually Works in Industry: Mechanisms, Patterns, and Dynamics
}

\author{
Franz Zieris
}

\begin{abstract}
During pair programming (PP), two software developers work closely together on a technical task on one computer. Practitioners expect a number of benefits, such as faster progress, higher quality, and knowledge transfer. Much of prior research focused on directly measurable effects from laboratory settings, but could not explain the large variations observed. My research follows the Grounded Theory Methodology and is aimed at understanding how PP actually works by uncovering the underlying mechanisms to ultimately formulate practical advice for developers. The main findings from my qualitative analysis of recordings of 27 industrial PP sessions are: Task-specific knowledge about the software system is crucial for pair programming. Pairs first make sure they have a shared understanding of the relevant parts before they acquire lacking knowledge together. The transfer of general software development knowledge plays a rather small role and only occurs after the pair dealt with its need for system knowledge. Pairs who maintain a shared understanding may have short, but highly-productive Focus Phases; if their Togetherness gets too low, however, a Breakdown of the pair process may occur.
\end{abstract}

\section{Introduction}

The idea of pair programming (PP) is probably as old as programming itself: Instead of dealing with a technical problem alone, two software developers sit down on one machine and work on the task together. In the 1990s, Coplien described this idea with his Pair Design pattern [6, p. 294], and it has become widely known under its current name in the context of Beck's agile development method eXtreme Programming [2], where it is one of the twelve core practices.

As Beck puts it, "pair programming is a dialog [...] a conversation at many levels" [2, p. 100]. It is a universal development practice that can be applied

\footnotetext{
F. Zieris $(\bowtie)$

Freie Universität Berlin, Berlin, Germany

e-mail: zieris@inf.fu-berlin.de
} 
to technical tasks beyond mere implementation, such as specifying requirements, architectural and lower-level design, debugging, and testing. It is independent of software domains and technology stacks. With screensharing or dedicated tools, the pair members do not even need to share the same physical workspace [11]. Consequentially, PP is widely used: Estimated conservatively, about $30 \%$ of all developers pair-program at least sometimes [13]. Surveys show that practitioners expect a range of benefits [3]:

- Two developers combine their knowledge to produce more ideas and work on more complicated tasks than either pair member could alone and produce better designs in less time.

- They build up lacking knowledge for debugging or system understanding faster and more reliably, which also helps them catch defects in the making.

- They may learn from each other or acquire new knowledge together, which helps them with future tasks and avoids knowledge silos in the team.

In industrial contexts, the transfer of knowledge is particularly important, which is why companies such as Pivotal (now part of VMware) use "continuous pair programming" and "overlapping pair rotation" for its long-term effects [12].

Although pair programming has been a research subject since the 1990s, it is still not clear to which degree and under which conditions it holds up to these expectations. Through my research, I want to provide actionable advice for practitioners by understanding how PP actually works, which prior research mostly failed to explain (see Sect. 2): Laboratory experiments disregard the complex process that is the pairs' interactions and rely on unrealistically small and simple tasks which do not come close to the knowledge demands of industrial software development. To close that gap, I collected data in industry-mostly video-recordings of everyday PP sessions, along with field observations and interviews - and analyzed it qualitatively (see Sect. 3). I provide a short overview of parts of my findings and how I validated them with practitioners in Sect. 4 before I conclude in Sect. 5. This chapter is based on four peer-reviewed publications [20-22, 24] and my PhD thesis [19].

\section{Overview of Pair Programming Research}

There are two main categories of pair programming studies. On the one hand are quantitative studies that compare, under controlled conditions, the results of developers working in pairs to those working alone (Sect. 2.1). Here, the pairs are often formed by researchers and work on carefully chosen tasks. On the other hand are qualitative analyses of natural situations where developers form pairs in a selfdecided, often spontaneous manner to work on complex issues (Sect. 2.2).

In the following discussion, I exclude the plethora of studies on pair programming in educational settings where the worked-on tasks are merely a means to an end such as homework assignments or small student projects. I also exclude 
PP studies that are based solely on questionnaires and interviews, as the subjects' reports do not necessarily reflect real events accurately and in detail.

\subsection{Quantitative Pair Programming Studies: Findings and Problems}

There has been a number of controlled experiments in which the quality and speed of programming pairs is compared to solos. The meta-analysis by Hannay et al. [7] covers 18 such experiments and finds small and medium positive PP effects on quality and (wall-clock) time, ${ }^{1}$ but no significant effect on effort: ${ }^{2}$ Pairs appear to be faster, but not twice as fast to outweigh the immediate cost of paying two developers. More noteworthy, however, is the large between-study variance, or heterogeneity, which indicates that the experiments actually measured different effects rather than the same effect with some added random noise. Future work should thus focus "on untangling the moderating factors" such as task complexity, developer experience (in programming and in pair-programming), motivation, and team climate [7].

The large experiment by Arisholm et al. [1] attempted to understand the influence of two of these factors on quality and time by hiring 295 professional software developers to work either alone or in pairs on a set of corrective maintenance tasks. To test the impact of developer experience, the groups were split according to their level of expertise (junior, intermediate, senior). Additionally, half of the subjects worked in either a "simple system" or a "complex" one in order to investigate the role of task complexity. The researchers identified a few tendencies (such as simple tasks get done faster with PP and juniors appear to produce higher quality with PP), but could not explain the moderating effect of either task complexity or experience because many differences between the respective subgroups were not statistically significant or even contradictory. In the words of the authors: "we are still far from being able to explain why we observe the given effects."

The recurring problems of many, even high-profile quantitative PP studies are:

1. There are simply too many potential moderator variables to be tested in controlled experiments with reasonable effort. The above experiment by Arisholm et al. [1] failed to understand just two and failed despite tremendous effort and cost.

2. The experimental situations differ from industrial practice in a number of relevant ways, including the following (illustrated with aspects of Arisholm et al.'s experiment): The subjects have little prior experience with the practice of working in pairs (93 out of 98 pairs had no prior PP experience); the systems

\footnotetext{
${ }^{1}$ Note that the $95 \%$ confidence intervals of the standardized effect sizes are quite large: Hedge's $g=[0.07,0.60]$ for the effect on quality and $g=[0.13,0.94]$ for time.

${ }^{2}$ The $95 \%$ confidence interval accordingly spans negative and positive values: $g=[-1.18,0.13]$
} 
are rather small (even the "complex system" comprised merely 12 classes and 287 lines of code); the tasks are small, too, and span only short time periods (the average pair completed all their three tasks in $1 \mathrm{~h}$ ); developers have no say in with whom to pair or whether to pair at all for the given task (researchers formed only pairs with the same level of expertise); common quality metrics only account for short-term effects (e.g., number of passed test cases, or just a single binary correctness value). Additionally, the subjects work in systems completely unknown to them, which does not at all represent everyday practice where developers usually build up a mental model of their system over months or even years.

All these constraints are in service of keeping measurements comparable between subjects and the extent of the experiments manageable. At the same time, they reduce the complexity of software development to mere programming contests - and are still not able to explain the observed effects.

3. In effect, pair programming is considered to be something canonical which developers simply do when told to do so. But how to pair-program "right"? Do the pair members discuss several ideas upfront and carefully select the best? Do they assume the often proposed roles of active "driver" who writes the code, and "navigator" who watches out for defects and larger implications (e.g., Williams and Kessler [17])? Or do they follow the idea of one partner until they hit a deadend and then switch places? Or does one partner remain silent until she notices a problem? Different pairs will probably follow different approaches.

Overall, quantitative comparisons of solo and pair programming in contrived settings based solely on summative metrics and short-term outcomes are of limited scientific value. To gain a deeper understanding of how and when PP actually works, the underlying process needs to be studied using qualitative research approaches that observe and analyze the practice under natural conditions in real industrial contexts.

\subsection{Qualitative Pair Programming Studies: Findings and Problems}

A few qualitative-quantitative studies recorded PP sessions in industrial contexts (e.g., [5]) or at least involved professional developers (e.g., [16]), transcribed and labeled the pairs' dialogs following some coding scheme, and then calculated different statistics. These studies show that there is constant communication during pair programming and that there are no systematic differences between the type of utterances of supposed "drivers" and "navigators"- at least for developers with more than 6 months of pair programming experience [4]. However, these studies use cumulative metrics which only reflect the total number of occurrences of certain events, but not their temporal and causal order. Whether a pair member does $X$ twice in the middle of the session, or once in the beginning and once again in the end- 
it is counted as two $X$ s either way. Without considering the process, such research approaches are also not well suited to explain pair programming.

Purely qualitative studies focus on building theories rather than testing hypotheses. They characterize PP processes on a conceptual level and show that good pairs follow certain communication patterns (e.g., explicit and implicit teaching [8], or Restarting, Planning, Action [18]). A central problem of many such studies is that they often result in loose collections of labels concerning some PP aspect, rather than aiming for an integrated theory of PP as a whole. To give one example, Plonka et al. [8] list six "teaching strategies" but do not investigate their relationships, e.g., how developers decide which one to use, whether there are preferred ones, or whether they are a matter of conscious decision at all. These studies do not provide obvious starting points for further research. Furthermore, a practitioner perspective—which would at least hint at possible applications in industry—is often missing.

\section{Research Goal, Data, and Method}

The goal of my research is to understand how pair programming, and in particular, knowledge transfer in pair programming, actually works. This is not about deciding whether pair programming is "better." Rather, I want to provide actionable advice for practitioners who want to pair-program, allowing them to avoid problems and reap the potential benefits.

My qualitative research follows the Grounded Theory Methodology (GTM) as formulated by Strauss and Corbin [14] and builds on the groundwork of Salinger and Prechelt [10], who developed a "vocabulary" of base activities to characterize the fundamental building blocks of a PP process.

The basis for my analyses is data which colleagues and myself have been collecting in industry since 2007. So far, we recorded 67 sessions of professional software developers in 13 companies working on their everyday tasks in pairs (sometimes also in groups of three or four). The recordings consist of a screencast, webcam video, and audio of the pair's dialog (see Fig. 1). We also conducted reflective interviews with the developers the day after their recording and organized group discussions and workshops to achieve a better understanding of the teams' contexts and to evaluate our findings. All data collection activities are detailed in a technical report [23].

In the manner of theoretical sampling [14], I iteratively selected material from the already available repository and established new contacts with three companies to collect additional data. For my analysis, I selected sessions with different contexts (e.g., application domains and technology stacks) and with developers at varying experience levels in both homogenous and heterogenous pair constellations. In total, I analyzed 27 sessions from 10 companies with 29 developers (see Table 1).

I did not perform my analyses on transcripts, but directly on the video material. Notes from field observations and reflective developer interviews provided 
Table 1 Analyzed sessions, some of which continue earlier ones $(\sqcup)$. Sessions JA1 and JA2 were distributed, all others colocated; MA1, OA1, OA2, OA5, and OA8 are in English, all others in German. Developers C4, C6, and O3 are female, all others are male. See [23] for more details

\begin{tabular}{|c|c|c|c|}
\hline ID & Length & Developers & Session content \\
\hline \multicolumn{4}{|c|}{ Company A: Content Management System (Java, Objective-C, SQL) } \\
\hline AA1 & $2 \mathrm{~h} 22 \mathrm{~min}$ & A1 A2 & $\begin{array}{l}\text { Fix five similar bugs touching both frontend and } \\
\text { backend }\end{array}$ \\
\hline \multicolumn{4}{|c|}{ Company B: Social Media (PHP, JavaScript, SQL, HTML, CSS) } \\
\hline BA1 & $1 \mathrm{~h} 46 \mathrm{~min}$ & B1 B2 & $\begin{array}{l}\text { Read foreign code, implement cache, discuss } \\
\text { specification }\end{array}$ \\
\hline BB1 & $1 \mathrm{~h} 21 \mathrm{~min}$ & B1 B2 & $\begin{array}{l}\text { New feature from scratch (template); discuss } \\
\text { requirements }\end{array}$ \\
\hline BB2 & $1 \mathrm{~h} 51 \mathrm{~min}$ & B1 B2 & $\begin{array}{l}\llcorner\text { impl. model, controller, template; discuss } \\
\text { requirements }\end{array}$ \\
\hline BB3 & $1 \mathrm{~h} 32 \mathrm{~min}$ & B1 B2 & $\begin{array}{l}\llcorner\text { implement template, controller; discuss } \\
\text { requirements }\end{array}$ \\
\hline
\end{tabular}

Company C: Graphical Geo Information System (Java)

\begin{tabular}{|c|c|c|c|}
\hline CA1 & $1 \mathrm{~h} 18 \mathrm{~min}$ & $\mathrm{C} 1 \mathrm{C} 2$ & Implement new form in GUI (C1 already started) \\
\hline CA2 & $1 \mathrm{~h} 24 \mathrm{~min}$ & C2 C5 & $\begin{array}{l}\text { Architecture discussion (C5 already started), } \\
\text { refactoring }\end{array}$ \\
\hline CA3 & $2 \mathrm{~h} 10 \mathrm{~min}$ & C6 C7 & $\begin{array}{l}\text { Implement context menu entry, incl. test case \& } \\
\text { refactoring }\end{array}$ \\
\hline CA4 & $1 \mathrm{~h} 34 \mathrm{~min}$ & $\mathrm{C} 4 \mathrm{C} 7$ & $\begin{array}{l}\text { Implement selection feature } \mathrm{w} / \text { special } \\
\text { key-binding }\end{array}$ \\
\hline CA5 & $1 \mathrm{~h} 23 \mathrm{~min}$ & C3 C4 & Implement feature to split graphical elements \\
\hline
\end{tabular}

Company D: Estate Customer Relationship Management (Java, XML)

\begin{tabular}{l|l|l|l} 
DA2 & $2 \mathrm{~h} 24 \mathrm{~min}$ & D3 D4 & Planned feature impl., turned to widespread
\end{tabular} refactoring

\begin{tabular}{|c|c|c|c|}
\hline \multicolumn{4}{|c|}{ Company E: Logistics and Routing $(C++, X M L)$} \\
\hline EA1 & $1 \mathrm{~h} 17 \mathrm{~min}$ & E1 E2 & $\begin{array}{l}\text { Step-by-step debugging of display error in the } \\
\text { GUI }\end{array}$ \\
\hline \multicolumn{4}{|c|}{ Company J: Data Management for Public Radio Broadcast (Java) } \\
\hline JA1 & $1 \mathrm{~h} 7 \mathrm{~min}$ & J1 J2 & $\begin{array}{l}\text { Walkthrough of J2's code, discuss possible } \\
\text { refactorings }\end{array}$ \\
\hline JA2 & $1 \mathrm{~h} 15 \mathrm{~min}$ & J1 J2 & Review of J2's new API, define requirements \\
\hline \multicolumn{4}{|c|}{ Company K: Real Estate Platform (Java, SQL, CoffeeScript) } \\
\hline KA1 & $1 \mathrm{~h} 59 \mathrm{~min}$ & K1 K2 & $\begin{array}{l}\text { Set up dev. env. discuss inter-system API } \\
\text { design, } 1 \text { st impl. }\end{array}$ \\
\hline KB1 & $53 \mathrm{~min}$ & $\mathrm{~K} 2 \mathrm{~K} 3$ & $\begin{array}{l}\text { Add new class to model, write and debug } \\
\text { database migration }\end{array}$ \\
\hline KC1 & $59 \mathrm{~min}$ & $\mathrm{~K} 2 \mathrm{~K} 3$ & $\begin{array}{l}\text { Set up test env., discuss test approaches for GUI } \\
\text { feature }\end{array}$ \\
\hline KC2 & $2 \mathrm{~h} 1 \mathrm{~min}$ & $\mathrm{~K} 2 \mathrm{~K} 3$ & 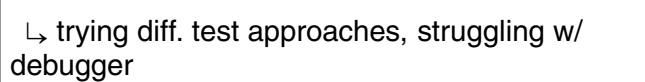 \\
\hline
\end{tabular}


Table 1 (continued)

\begin{tabular}{c|l|l|l}
\hline ID & Length & Developers & Session content \\
\hline Company M: Data Analysis in Energy and Transportation Sector (SQL) \\
\hline MA1 & 25 min & M1 M2 & \multicolumn{2}{l}{ Explanation of table model } \\
\hline Company O: Online Project Planning (CoffeeScript)
\end{tabular}

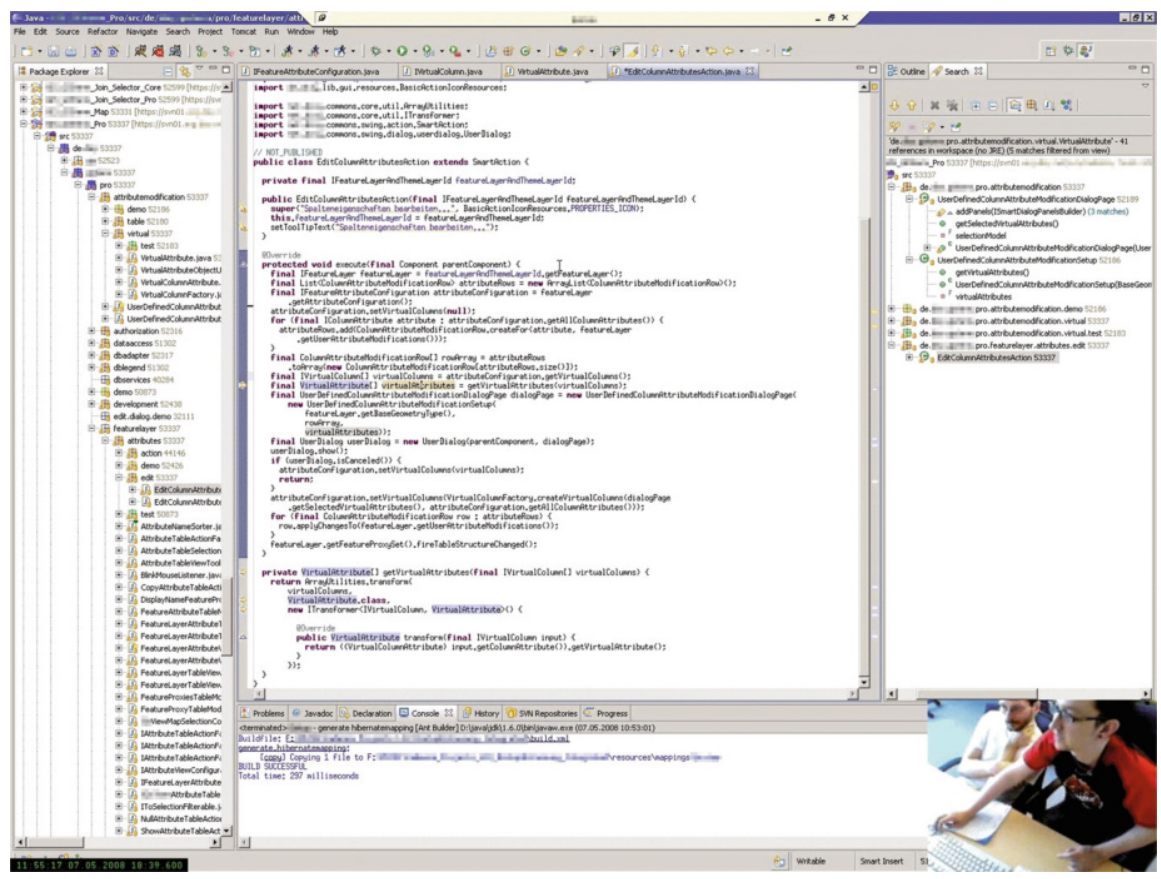

Fig. 1 Still frame of a recorded pair programming session (webcam video in bottom right corner) 
Table 2 Member reflection activities with their format and roles of participants (SM-Scrum Master, PO—Product Owner, TM-Technical Manager, D—Developer)

\begin{tabular}{l|l|l}
\hline Context & Format & Participants \\
\hline Company K & Presentation and discussion & $\sim 50$ (div. roles) \\
\hline Company O & Workshop & $2 \times \mathrm{SM}, 1 \times \mathrm{PO}$ \\
\hline Company P & 1 -on-1 interview & $1 \times \mathrm{SM}$ \\
\hline Company P & Workshop & $6 \times \mathrm{D}, 1 \times \mathrm{SM}, 1 \times \mathrm{PO}$ \\
\hline Company P & Post-recording interview $(3 \times)$ & $2 \times \mathrm{D}$ per interview $(3 \times \mathrm{D}$ in total) \\
\hline Company Q & 1-on-1 interview & $1 \times \mathrm{TM}$ \\
\hline Company R & Workshop & $10 \times \mathrm{D}, 2 \times \mathrm{TM}, 1 \times \mathrm{SM}$ \\
\hline
\end{tabular}

additional context information. I applied the GTM practices of open, axial, and selective coding [14] by starting at the level of thousands of individual utterances, and working my way up over hundreds of knowledge transfer episodes (running between a couple of seconds and a few minutes), to clusters of episodes, and to the overall dynamic of whole sessions, until my concepts reached theoretical saturation [14].

I evaluated my findings through different member reflection activities [15]: 1-on-1 interviews with Scrum Masters and workshops consisted of a short presentation of my research findings followed by reflections on the participants' personal experiences; post-recording interviews were more concrete, as I framed the developers' own most recent PP experience in terms of my concepts. Through the activities summarized in Table 2, I made sure my concepts and observations resonate with the practitioners. In the following section, I provide a brief overview of parts of my findings.

\section{Results: How Does Pair Programming Work?}

Here, I present two types of findings. In Sect. 4.1, I describe low-level differences in the way how pairs interact and work together. Although my research was originally only focused on knowledge transfer, deciphering these basic mechanisms and behavioral patterns occurred to me as relevant for understanding all higher-level aspects of pair programming.

I then skip over a few conceptual layers and take a bird's-eye view on pair programming sessions as whole in Sect. 4.2. I characterize how available knowledge and knowledge gaps affect developers in a pair situation and investigate the general dynamics that arise from this.

Section 4.3 then summarizes concrete ideas for how software development teams can apply my findings in their daily routine. 


\subsection{Fluency and Togetherness}

Among the analyzed pairs, I noticed considerable differences in how fluent their respective processes were. Most pairs have a continuous flow of conversation where they introduce new topics (which I mark with a $\star$ ), refer back to these topics to evaluate or extend them (marked with either $\Delta$ or 4 , in case the partner has yet to "arrive" at the same topic), and clear up the occasional misunderstanding through meta-communication ( - just like in any normal conversation. But a pair's Fluency can also take one of two extreme forms.

On the one hand, some pairs have high-paced Focus Phases during which there are practically no speech pauses and where the partners complete each other's thoughts, sentences, and even code lines: There is a quick succession of $\star s$ and $\Delta \mathrm{s}$. The developers may speak at the same time and in incomplete sentences, but nevertheless understand each other perfectly, such that corrective Os are rare. It is impossible to grasp the dynamic of a Focus Phase by reading a transcript alone. As an approximation, I compiled all the activities of one Focus Phase in Fig. 2: Within 60 seconds, the pair discusses 11 (!) different topics, constantly talking and editing.

On the other hand, the pair process may also suffer from a Breakdown, where the developers do not evaluate each other's ideas and proposals (more self-referential $<\mathrm{s}$ than pair-referential $\Delta s$ ) or may even fall into embarrassed silence (a communicative defect, marked with $\diamond)$ :

O3: "Ah, it expects a number, but we pass an object."

O4: [looks puzzled at O3]

O3: "It's an object, it's a key-value pair."

O4: [silently moves mouse cursor aimlessly across the screen for 30 seconds]

The Fluency of a PP process comes from the ease with which the two developers can understand the content and intentions of each other's actions and utterances. I call this a pair's momentary Togetherness. Through analyzing situations of both high and low Fluency, of close and little Togetherness, I identified the following five contributing factors:

1. A shared understanding of the software system allows for efficient communication (e.g., by speaking of "Factory" instead of "FeatureLayerAttributeTableCellRendererFactory" and still be perfectly understood by the partner). Where such shared understanding is lacking, more wordy explanations are necessary or more misunderstandings need to be cleared up.

2. A shared understanding of software development in general has a similar influence. Knowing about common architectural styles, design patterns, and typical ways to approach certain problems allows for efficient communication and discussion of ideas. Otherwise, it is more difficult to evaluate the partner's proposals which puts a strain on a pair's Togetherness (e.g., "Erm, okay? Go on then. I'm quite out of my depths here.").

3. One shared plan, e.g., in the form of a strategic decision made earlier, provides a backdrop for quickly understanding and amending tactical proposals: 


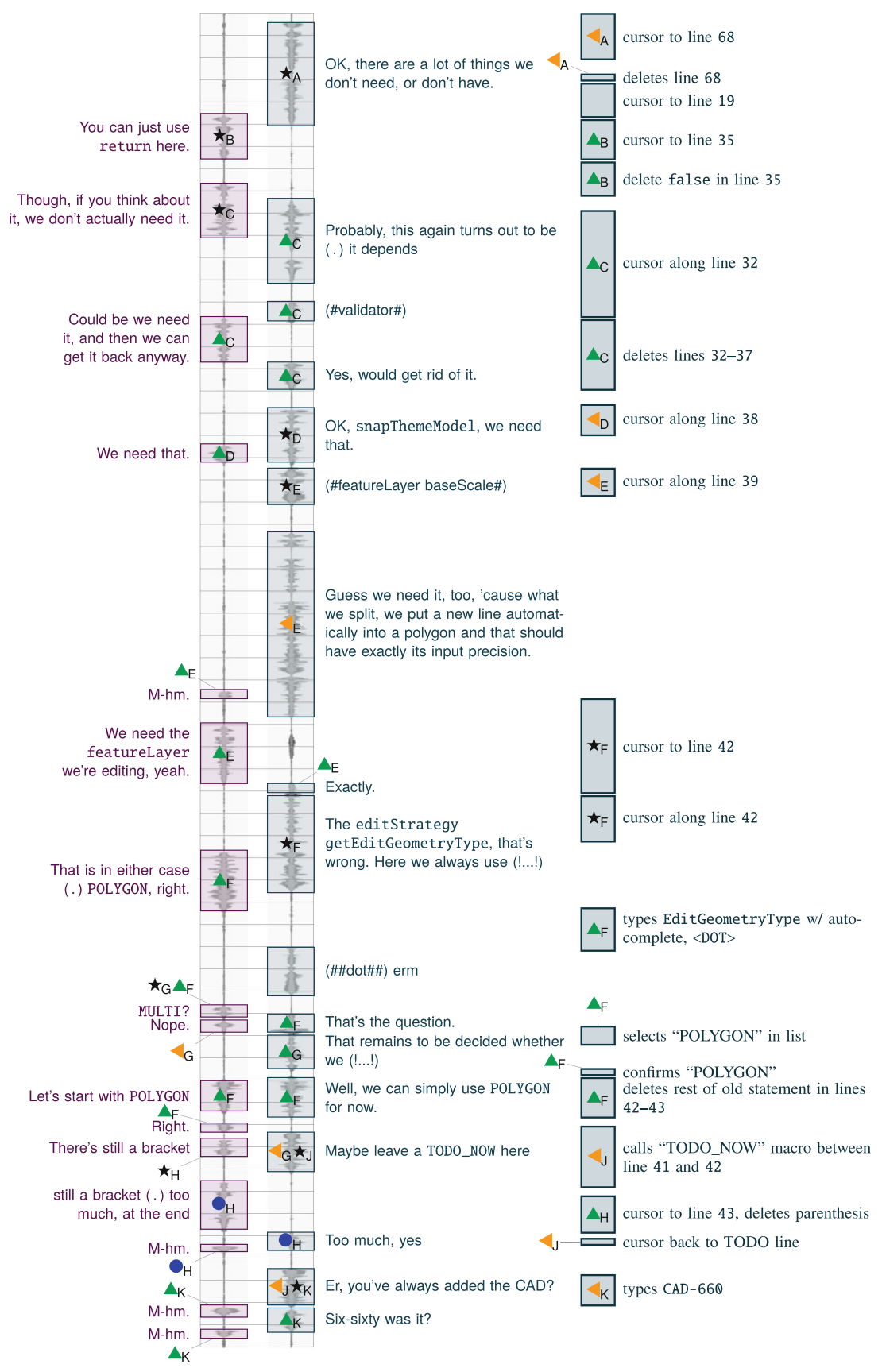

Fig. 2 A Focus Phase of 60 seconds (time runs from top to bottom; columns 1 and 2 contain the pair's utterances, and column 3 the editing activities). The pair starts new topics, furthers them, and repairs potential misunderstandings in quick succession. Overall, they discuss eleven topics (indices $\mathrm{A}-\mathrm{K}$ ), while one partner performs code changes on-the-fly 
C3: "Shall we copy the code and check line-by-line whether it makes sense?"

C4: "OK, let's see what we need."

[... two minutes later ...]

C3: "[opens file] $\mathrm{Ok}$, there are lot of things we don't need."

C4: [pointing at existing code] "You can just use return here."

Without such an understanding, misunderstandings are more common because one developer may have difficulties understanding how their partner's actions fit in the big picture.

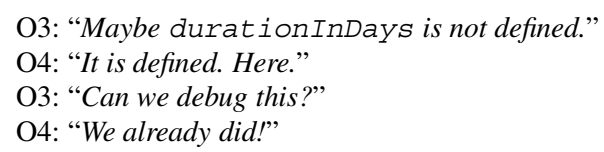

4. Workspace awareness is reduced in distributed pair programming due to the spatial separation of the two developers, but can also be a problem in colocated settings, e.g., with too-small font sizes ("Where are you right now, which class?").

5. A language barrier can come from using a foreign language (e.g., in a discussion of two non-native English speakers: "An 'offset' is a duration?") and from idiosyncrasies in one's way of speaking (e.g., saying "Wait a second" while meaning "I'm now going to take mouse and keyboard.").

The following short exchange illustrates how several of these factors come together.

\section{A Pair's Moment of High Togetherness}

The pair agreed on changing the signature of an interface method, and developer C5 asks his partner C2:

C5: "Erm, do you know the-" [right-clicks on method name to open context menu]

"How I access the feature to change a method?"

$\mathrm{C} 2$ : "That doesn't get you anywhere."

While C2 does not answer C5's question, his reaction reveals that he very well understood what his partner intends to do. In particular, four factors help C2 understand the following aspects, allowing him to react so promptly:

- No language barrier: C5's utterance is a question to which he expects an answer.

- Workspace awareness: C5 wants to apply a refactoring (opened context menu) on some method (cursor position).

- Shared understanding of software development: C5 refers to the refactoring "Change Method Signature," which is known to both developers for changing a method's signature in all places where it is declared, implemented, or called. 
- One shared plan: C5 wants to apply this particular refactoring probably with the expectation to save some manual editing on their way to change the interface.

This exchange also illustrates that high Togetherness is not the same as agreeing with the partner: $\mathrm{C} 2$ apparently disagrees with the C5's assessment of how much time and effort can be saved by applying the automatic refactoring.

The five factors are not independent from another and problems from one factor may also add to the difficulties of another (e.g., a language barrier and reduced workspace awareness). Good pairs become aware of their shortcomings and find ways to cope with them, e.g., compensating reduced workspace awareness by more verbose commentary of their editing activities (factor 4), or taking the time to explain some programming idiom which puzzled the partner (factor 2). A pair's Togetherness may change over the course of a session. The partners may (inadvertently or consciously) reduce their Togetherness ("You go on. I say when I'm back on track.") or maintain their Togetherness through repair activities ("Why did you just do this?").

The not-explained variance of the effects measured in PP experiments (see Sect. 2.1) could be due to differences in Togetherness among the subject pairs and different competencies to deal with them. Factors 2 and 3 (shared understanding of software development and one shared plan) may play an important role even in contrived experimental situations. But there is no telling in hindsight. This observation emphasizes the importance of understanding the underlying processes rather than just reducing PP to time spent and lines of code produced. With such a narrow perspective, pair properties such as Fluency and Togetherness remain hidden.

\subsection{Knowledge Wants, Knowledge Needs, and Prototypical Dynamics}

At the heart of my research is the question of how pair programmers transfer and acquire knowledge. A central observation is that knowledge transfer happens in every session — be it as the decided goal to introduce the partner to a part of the system she has never seen before, or as a side-effect of closing a gap in each other's system or programming understanding (maintaining Togetherness, see Sect. 4.1).

In the course of a pair programming session, a developer will sooner or later perceive a Knowledge Want, which could be paraphrased as I want to know this (internal Knowledge Want), I want you to know this (external), or We want to know this (collective). This is what motivates episodes of knowledge transfer during 
Fig. 3 Initial constellations of Knowledge Needs in the analyzed pair programming sessions. One-sided $S$ gap and Two-sided $S$ gap are quite common ("normal" software development); No relevant gaps and Too-large, two-fold gap are rare (greenfield development, and impossible task); Complementary gaps can lead to a mutually satisfying teaching and learning experience

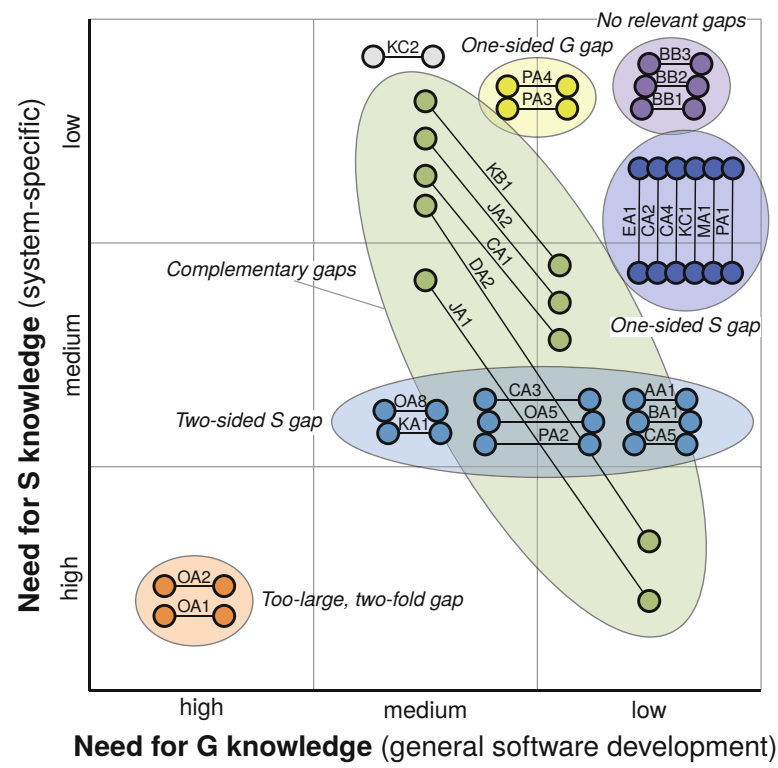

which she asks her partner, provides her with explanations, or involves her in the acquisition of new knowledge through reading source code until her Knowledge Want is satisfied.

In contrast to local Knowledge Wants, a developer is not necessarily aware of the extent of her global Knowledge Need, which are her knowledge gaps regarding to the specific demands of the task. The same task may be easy for someone with all the relevant knowledge available (low Knowledge Need), or rather difficult if the system or technology are unfamiliar (high Knowledge Need). Conversely, the same developer will have to deal with different Knowledge Needs for different tasks.

Based on the analysis of hundreds of knowledge transfer episodes, I distinguish two types of knowledge that are relevant in industrial pair programming sessions: System-specific $\mathbf{S}$ knowledge, which includes knowledge about requirements, the architecture and low-level design, concrete usage of technologies, and behavior of the system and defects; and generic $\mathbf{G}$ knowledge about software development as such, which covers design patterns, programming languages, tools, and libraries.

Pair programming sessions can now be characterized by the extent of the partners' initial Knowledge Needs regarding the S- and the G-dimension and the trajectory they take by pursuing their Knowledge Wants. I identified six recurring initial pair constellations (Fig. 3), including pairs with Complementary Gaps, where one partner possesses more task-relevant $\mathrm{G}$ knowledge (e.g., about design patterns and refactorings) while the other has more task-relevant $\mathrm{S}$ knowledge (e.g., because she originally implemented that part of the system). Others may have a One-Sided $S$ Gap, which occurs often when one partner built up an $\mathrm{S}$ advantage through prior work. 
Fig. 4 Three session dynamic prototypes. Pairs first address differences in system understanding (Primary Gap) before acquiring lacking system understanding together (Secondary Gap). A difference in software development knowledge then poses an Opportunity

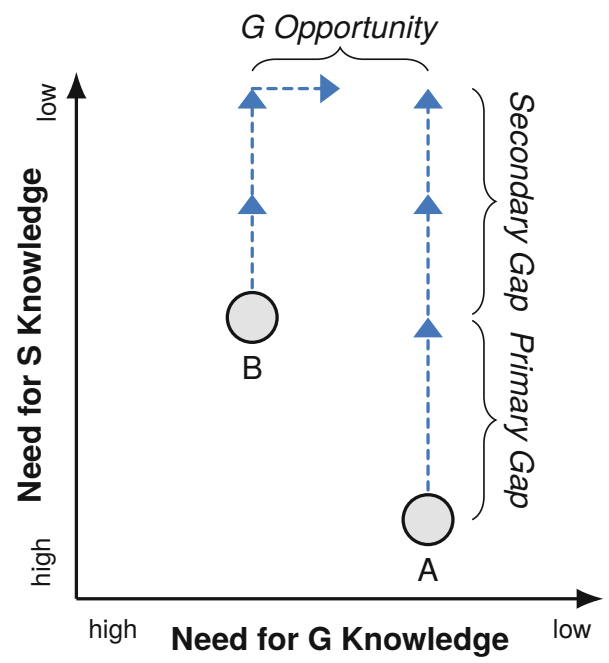

Considering the knowledge trajectories taken by the pairs in all sessions, I discovered the following three prototypical session dynamics (see also Fig. 4):

1. Closing the Primary Gap, which is the difference in task-specific system understanding between the pair members. There might be no such gap, if both developers worked on the task before. Quite often, however, a developer joins another who already worked on the task. This is the case in this example, where one developer has a fresh mental model of the system and his partner does not:

C1: "I already started and programmed for an hour. I started with the GUI. I'll show you quickly" [opens overview of recent changes]

In another scenario, one partner's last encounter with a piece of software was several months ago, but he still has an $\mathrm{S}$ knowledge advantage because his partner has never seen it:
J2: "Do you know the plugin, or don't you know it?"
J1: [exhales audibly] "Just show to it me again."
$\mathrm{J} 2$ : "OK, I can give you the big picture of what this plugin does, overall."

If there is a Primary Gap, pairs address it early in their session. Not dealing with this gap properly is an impediment to the pair's Togetherness (factor 1, shared system understanding, see Sect. 4.1).

2. Closing the Secondary Gap, which consists of acquiring task-relevant system knowledge which neither developer possesses. This is common in debugging scenarios but can also happen in the context of feature implementations where the pair needs to find the right spot to add new logic. Pair programmers may acquire such understanding together by reading source code or using a debugger. Good pairs make sure to maintain their Togetherness in this phase, too: If one partner pulls ahead, she helps her partner take the ental steps. Note that in some 
situations, it may not be necessary for both partners to understand the system to the same degree, e.g., if only one of them is expected to continue working on it in the future.

3. A G Opportunity arises when one partner possesses more $G$ knowledge than the other. Unlike a difference in $\mathrm{S}$ knowledge, a $\mathrm{G}$ knowledge difference was never a problem in the analyzed sessions. In all instances, however, pairs only seized this opportunity after closing their Primary and Secondary Gaps. Even if the Knowledge Need was already there, pairs do not appear to develop a Knowledge Want regarding G knowledge until their S Knowledge Wants are satisfied. Therefore, exchanges like this one tend to happen only late in the session:

[... 1:30 hours into session DA2 ...]

D4: "Do you know about OSGi class loading?"

D3: "Class-what? Not really, no."

D4: "Should I tell you?"

D3: "Sure."

These three dynamics characterize the majority of all analyzed PP sessions: Pairs first address their Primary Gap, then their Secondary Gap, before potentially seizing their G Opportunity.

The special case are pairs where both partners lack relevant $\mathrm{G}$ knowledge. A moderate deficit is not problematic, as the developers simply try to complete their task with the means available. If the pairs lack a lot of G knowledge, however, their task gets much more difficult because they cannot build up the necessary system understanding, as one developer exclaims after an hour of trying to understand a single source file:

\section{O3: "Type? Function? I don't even know what this is!"}

Such situations should be rare, though, as practitioners appear to avoid such massive overtaxation by choosing partners and tasks that match their existing knowledge.

Overall, knowledge transfer in pair programming in industry is mostly about system understanding. Not only does the vast majority of knowledge transfer episodes pertain to system-specific $S$ knowledge rather than generic $G$ knowledge; pair programmers also deal with their $\mathrm{S}$ knowledge gaps first, before they may look for $G$ Opportunities to explain general software development concepts.

\subsection{Practical Applications}

I developed the following three practices for software development teams to make use of my findings in their everyday practice:

1. Pair Forming: Teams can use the G-S chart (Fig. 3) to discuss the Knowledge Needs of all team members for an upcoming task and find a favorable constellation, e.g., one with a $G$ Opportunity. 
2. Set Session Goal: Do both pair members need to understand the task-relevant system parts enough to continue the work, or is some Primary Gap tolerable? Is there a G Opportunity that could be seized?

3. Reflect on Session: After the session, the pair may consider questions like: Did we underestimate our Knowledge Needs, i.e., are there lacking pieces of S or G knowledge whose relevance we were not aware of? Should we discuss these with the rest of the team?

I evaluated my findings and the practice ideas in four companies through workshops and interviews. Overall, concepts presented in this chapter were well received by the practitioners. Due to practical constraints such as team size, developer availability, and tasks with a rigid scope, I was only able to test the third practice Reflect on Session in the field. It helped the developers remember and appreciate pieces of knowledge that they transferred or acquired in their session:

P1: "I guess that [P3] learned in both dimensions, even technologically [G knowledge] since we now use more PHP7_"

P3: "Right! I totally forgot about that. That was actually really cool."

Although I had no chance to observe it, the practitioners also expected the other two practices (Pair Forming and Set Session Goal) to have positive effects, if their context allowed them more degrees of freedom.

\section{Summary and Outlook}

Pair programming is a way of developing software that is amendable to every aspect of software development in all application domains, regardless of concrete technology. It is widely used in industry with the expectation to speed up development, lead to higher quality, and spread knowledge in development teams. Prior research focused too much on directly measurable effects and too little on the processes and mechanisms that are at work when two software developers tackle a complex problem together in a real environment. My qualitative analysis of 27 industrial pair programming sessions from 10 companies lead to the following central observations:

- Despite what has been proclaimed for many years, the typical programmer pair in industry does not consist of an active "driver" and a strategically thinking and defect-hunting "navigator," but of two software developers who are engaged in a dialog, discussing ideas and building up a shared understanding of task and system. Admittedly, one of them usually does happen to use keyboard and mouse to interact with the computer for some time span, but that is not what makes pair programming work.

- What allows pairs to a have fluent process is a high degree of Togetherness which enables them to understand each other's ideas and arguments. In particular, pairs need to maintain a shared understanding of their software system and of 
software development in general. Having one shared plan also helps a long way. Additionally, pairs may need to cope with reduced workspace awareness or language barriers of sorts. Doing all of the above may lead to short, but highly productive Focus Phases; if their Togetherness drops too low, a Breakdown of their process may ensue.

- Two main types of knowledge are relevant in industrial pair programming sessions: Pairs mostly deal with (lacks of) specific knowledge about their software system ( $S$ knowledge) and to a lesser degree with (lacks of) generic knowledge about software development general ( $G$ knowledge). Transfer of S knowledge practically happens in all pair programming sessions.

- There is a number of recurring pair constellations which result from the individually different levels of applicable pre-existing knowledge. A pair's constellation is highly task-dependent: The same two developers may have their supposed "expert" and "novice" labels reversed when they work in different parts of the system or happen to need certain technology skills. Amending the task's scope therefore can put both developers in the position to teach the other something new.

My work focused primarily on two of the five factors that influence a pair's Togetherness: Their shared understanding of the software system and of software development in general (factors 1 and 2, see Sect. 4.1). Other research by Schenk et al. [11] already looked at how good pairs deal with reduced workspace awareness (factor 4). This leaves an in-depth analysis of decision making, i.e., how pairs come up with one shared plan (factor 3), as important future work. Although I did not encounter it in my data, practitioner literature suggests that they are also more ways for a pair's process to have a Breakdown, e.g., due to power dynamics [9].

I was personally involved in collecting primary data in four companies $(\mathrm{K}, \mathrm{M}, \mathrm{O}$, and P; see also Table 1) and evaluated the above findings in five contexts (Table 2), where they were met with great resonance, which is an important quality criterion of qualitative research besides the rigorous analysis of rich data [15]. Beyond the "scientific" results already discussed, my interactions with the practitioners were fruitful, because it made the teams aware of how little thought they had previously put into what their implementation of the pair programming practice actually entails:

- Company O, for example, considered themselves an "all-PP company." They could not organize a structured onboarding process and mandated pair programming and regular team rotations instead. However, during my 4-week observation period, there were only a few PP sessions, but a widespread lack of technology knowledge, which the Scrum Masters only became aware of through my research activities.

- The product owner and developers in company $\mathrm{P}$ expected pair programming to yield "all the benefits," but had no priority: Better design, fewer defects, faster progress, transfer of knowledge, and enjoyable work were all equally important, and each PP session was expected to have these effects. My workshop made them reconsider their priorities. 
It is my impression that my discussions with the practitioners made them reflect on their work, motivated discussions on which PP effects they actually care about, and made them see forming pairs considerately, setting sessions goals, and reflecting on recent PP sessions as opportunities to achieve these effects.

I try to keep in touch with the companies even years after the initial data collection (in particular through their Scrum Masters) and continue to seek the exchange with more practitioners to make my findings more widely known.

\section{References}

1. Arisholm, E., Gallis, H., Dybå, T., Sjøberg, D.I.: Evaluating pair programming with respect to system complexity and programmer expertise. IEEE Trans. Softw. Eng. 33(2), 65-86 (2007). https://doi.org/10.1109/TSE.2007.17

2. Beck, K.: Extreme Programming Explained: Embrace Change. Addison-Wesley, Reading (1999)

3. Begel, A., Nagappan, N.: Pair programming: What's in it for me? In: Proceedings of the Second ACM-IEEE International Symposium on Empirical Software Engineering and Measurement, pp. 120-128. ACM, New York (2008). https://doi.org/10.1145/1414004.1414026

4. Bryant, S.: Double trouble: Mixing qualitative and quantitative methods in the study of extreme programmers. In: IEEE Symposium on Visual Languages and Human Centric Computing, VL/HCC '04, pp. 55-61. IEEE, Piscataway (2004). https://doi.org/10.1109/VLHCC.2004.20

5. Bryant, S., Romero, P., du Boulay, B.: Pair programming and the mysterious role of the navigator. International Journal of Human-Computer Studies 66(7), 519-529 (2008). https:// doi.org/10.1016/j.ijhcs.2007.03.005

6. Coplien, J.: A generative development-process pattern language. In: Rising, L. (ed.) The Patterns Handbook: Techniques, Strategies, and Applications, pp. 243-300. Cambridge University Press, Cambridge (1998)

7. Hannay, J.E., Dybå, T., Arisholm, E., Sjøberg, D.I.: The effectiveness of pair programming: a meta-analysis. Inform. Softw. Technol. 51(7), 1110-1122 (2009). https://doi.org/10.1016/j. infsof.2009.02.001

8. Plonka, L., Sharp, H., van der Linden, J., Dittrich, Y.: Knowledge transfer in pair programming: an in-depth analysis. International Journal of Human-Computer Studies 73, 66-78 (2015). https://doi.org/10.1016/j.ijhcs.2014.09.001

9. Pyhäjärvi, M.: Power dynamics in pairs and mobs (2018). https://visible-quality.blogspot.com/ 2018/05/power-dynamics-in-pairs-and-mobs.html

10. Salinger, S., Prechelt, L.: Understanding Pair Programming: The Base Layer. Books on Demand (2013). http://www.inf.fu-berlin.de/inst/ag-se/pubs/SalPre13-baseconbook.pdf

11. Schenk, J., Prechelt, L., Salinger, S.: Distributed-pair programming can work well and is not just distributed pair-programming. In: Proceedings of the 36th International Conference on Software Engineering, ICSE '14, pp. 74-83. ACM Press, New York (2014). https://doi.org/10. $1145 / 2591062.2591188$

12. Sedano, T., Ralph, P., Péraire, C.: Sustainable software development through overlapping pair rotation. In: Proceedings of the 10th ACM/IEEE International Symposium on Empirical Software Engineering and Measurement, ESEM '16, pp. 19:1-19:10. ACM Press, New York (2016). https://doi.org/10.1145/2961111.2962590

13. StackOverflow: 2018 stack overflow developer survey (2018). https://insights.stackoverflow. com/survey/2018

14. Strauss, A., Corbin, J.: Basics of Qualitative Research. Grounded Theory Procedure and Techniques. Sage Publications, New York (1990) 
15. Tracy, S.J.: Qualitative quality: eight "big-tent" criteria for excellent qualitative research. Qual. Inq. 16(10), 837-851 (2010). https://doi.org/10.1177/1077800410383121

16. Walle, T., Hannay, J.E.: Personality and the nature of collaboration in pair programming. In: Proceedings of the 3rd International Symposium on Empirical Software Engineering and Measurement, pp. 203-213. IEEE, Piscataway (2009). https://doi.org/10.1109/ESEM.2009. 5315996

17. Williams, L., Kessler, R.R.: Pair Programming Illuminated. Addison-Wesley, Reading (2002)

18. Zarb, M., Hughes, J., Richards, J.: Industry-inspired guidelines improve students' pair programming communication. In: Proceedings of the 18th ACM Conference on Innovation and Technology in Computer Science Education, pp. 135-140 (2013). https://doi.org/10.1145/ 2462476.2462504

19. Zieris, F.: Qualitative analysis of knowledge transfer in pair programming. Ph.D. Thesis, Fachbereich Mathematik und Informatik, Freie Universität Berlin (2020). https://doi.org/10. 17169/refubium-28718

20. Zieris, F., Prechelt, L.: On knowledge transfer skill in pair programming. In: Proceedings of the 8th ACM/IEEE International Symposium on Empirical Software Engineering and Measurement, ESEM '14, pp. 11:1-11:10. ACM, New York (2014). https://doi.org/10.1145/ 2652524.2652529

21. Zieris, F., Prechelt, L.: Observations on knowledge transfer of professional software developers during pair programming. In: Proceedings of the 38th International Conference on Software Engineering Companion, ICSE '16 (SEIP), pp. 242-250. ACM, New York (2016). https://doi. org/10.1145/2889160.2889249

22. Zieris, F., Prechelt, L.: Explaining pair programming session dynamics from knowledge gaps. In: Proceedings of the 42nd International Conference on Software Engineering, ICSE '20, pp. 421-432. ACM, New York (2020). https://doi.org/10.1145/3377811.3380925

23. Zieris, F., Prechelt, L.: PP-ind: Description of a repository of industrial pair programming research data (2020). https://arxiv.org/abs/2002.03121v5

24. Zieris, F., Prechelt, L.: Two elements of pair programming skill. In: Proceedings of the 43rd International Conference on Software Engineering, ICSE '21 (NIER). IEEE, Piscataway (2021). https://arxiv.org/abs/2102.06460

Open Access This chapter is licensed under the terms of the Creative Commons Attribution 4.0 International License (http://creativecommons.org/licenses/by/4.0/), which permits use, sharing, adaptation, distribution and reproduction in any medium or format, as long as you give appropriate credit to the original author(s) and the source, provide a link to the Creative Commons license and indicate if changes were made.

The images or other third party material in this chapter are included in the chapter's Creative Commons license, unless indicated otherwise in a credit line to the material. If material is not included in the chapter's Creative Commons license and your intended use is not permitted by statutory regulation or exceeds the permitted use, you will need to obtain permission directly from the copyright holder.

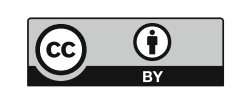

